\title{
Axisymmetric landslides on top-shaped asteroids
}

\author{
Deepayan Banik ${ }^{1}$, Kumar Gaurav ${ }^{2}$ and Ishan Sharma ${ }^{2}$ \\ ${ }^{1}$ Department of Aerospace Engineering, Indian Institute of Technology, Kanpur, UP, 208016,India \\ ${ }^{2}$ Department of Mechanical Engineering, Indian Institute of Technology, Kanpur, UP, 208016, India
}

\begin{abstract}
.
Spin rates of minor planets or asteroids are known to have been affected by several agents including but not limited to tidal fly-bys, impacts and solar radiation. Surface processes like landslides occur as a result of such rotational changes. We study the evolution of landslides on top-shaped rubble pile asteroids like 101955 Bennu and 162173 Ryugu, with the underlying core modeled as two solid cones fused back to back. Using a depth averaged avalanche theory applicable to granular flows we solve for axisymmetric landslides occurring at various spin rates and regolith friction. Static regions on the surface corresponding to different spin rates are identified from an equilibrium analysis. We then solve for landslides initiated at different latitudes. It is found that landslides equilibrate at lower latitudes as the spin rate is increased. Beyond a critical spin rate regolith is shed from the equator. This critical spin is higher for a lower value of the semi-apex angle of the cone.
\end{abstract}

\section{Introduction}

In the geophysical context, the length scale of surface flows may match the terrestrial scale of planetary bodies, for example, oceanic flows on the Earth and granular avalanches on rubble-pile asteroids like Bennu [1], Itokawa and Ryugu. However, the latter involves more complications: new terms in the momentum balance equations, a non-uniform gravity field and an undulating basal topography.

It is known that asteroids smaller than the kilometer scale but larger than $150 \mathrm{~m}$ have a lower limit to the observed spin period of about 2.1 hours. The structure of such asteroids is defined by a solid core with a layer of regolith on the surface [2]. In this direction [3] explores flow of regolith over an elliptical central body in a twodimensional setting. Here we extend that to three dimensions and study the flow of regolith down a rotating and gravitating top-shaped object resembling asteroids Bennu and Ryugu (Fig. 1). We include the effects of a varying gravity and basal curvature and use the principles of continuum mechanics to do so. To simplify the analysis we assume that the flow is shallow which allows us to average the governing equations along the depth of the flow.

\section{Theory for shallow granular flow over a top-shaped geometry}

We model a top-shaped body by fusing two cones along their base; see Fig. 2. A conical coordinate system is employed, with the basis vectors pointing down the slope, normal to it and in the azimuthal direction. Assuming axisymmetry simplifies our equations greatly and also prevents landslides from throwing the asteroid out of a pure spin state.

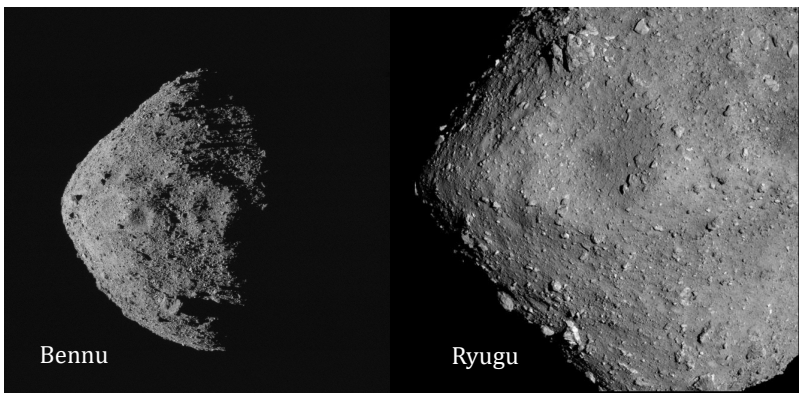

Figure 1. Images of top-shaped asteroids Bennu (left) and Ryugu (right) taken by NASA's OSIRIS-REx and JAXA's Hayabusa-II asteroid missions, respectively.

We next extend the theory of shallow granular avalanches developed in [4] to study landslides on the spinning double-cone top.

\subsection{Balance laws}

Here we present a overview of the mathematical treatment for shallow granular flows. The detailed derivation may be found in [5]. The mass balance equation for a bulk of incompressible flowing regolith is given by

$$
\nabla \cdot \boldsymbol{u}=0
$$

where, $\boldsymbol{u}$ is the flow velocity. The momentum balance in a frame of reference rotating with an angular velocity $\mathbf{\Omega}$ and angular acceleration $\boldsymbol{\alpha}$ is

$$
\rho_{\circ}\left[\frac{\partial \boldsymbol{u}}{\partial t}+(\boldsymbol{u} \cdot \nabla) \boldsymbol{u}\right]=-\nabla \cdot \boldsymbol{P}+\rho_{\circ}[\boldsymbol{a}-2 \boldsymbol{\Omega} \times \boldsymbol{u}-\boldsymbol{\alpha} \times \boldsymbol{r}],
$$


where, $\boldsymbol{u}$ is the velocity field, $\boldsymbol{P}$ is the pressure tensor (negative of the stress tensor), $\boldsymbol{r}$ locates a point on the surface with respect to the center of the body, and $\boldsymbol{a}$ is the effective gravity vector, the normal and downslope components of which are given by, respectively,

$$
\begin{aligned}
& a_{n}=\Omega^{2} \tau \cos \zeta-G(s) \sin (\zeta+\eta) \\
& \text { and } a_{s}=\Omega^{2} \tau \sin \zeta-G(s) \cos (\zeta+\eta),
\end{aligned}
$$

with $\eta$ being the angle made by the gravity vector $\boldsymbol{G}$ with the global vertical $(\mathrm{OZ}), \zeta$ is the semi-apex angle, $G(s)$ is the magnitude of the gravity, and $\tau$ and $s$ are the distances measured from the symmetry axis $\mathrm{Z}$ and the apex of the top-shaped body respectively.

\subsection{Coriolis and Euler forces}

Considering that our asteroid rotates about the $Z$ axis, the angular velocity vector in the curvilinear coordinates is given by

$$
\boldsymbol{\Omega}=-\Omega_{0} \cos \zeta \hat{\boldsymbol{e}}_{\boldsymbol{s}}+\Omega_{0} \sin \zeta \hat{\boldsymbol{e}}_{\boldsymbol{n}}
$$

The Coriolis and Euler acceleration terms are, respectively,

$$
\begin{gathered}
2(\boldsymbol{\Omega} \times \boldsymbol{u})=-2 \Omega_{0} v \sin \zeta \hat{\boldsymbol{e}}_{\boldsymbol{s}}+2(\cos \zeta w \\
+\sin \zeta u) \hat{\boldsymbol{e}}_{\boldsymbol{\phi}}-2 \Omega_{0} v \cos \zeta \hat{\boldsymbol{e}}_{\boldsymbol{n}} \\
\text { and } \boldsymbol{\alpha} \times \boldsymbol{r}=-\tau \alpha \hat{\boldsymbol{e}}_{\boldsymbol{\phi}},
\end{gathered}
$$

where, the $u, v$ and $w$ are components of the velocity along $\hat{\boldsymbol{e}}_{\boldsymbol{s}}, \hat{\boldsymbol{e}}_{\phi}$ and $\hat{\boldsymbol{e}}_{\boldsymbol{n}}$, respectively.

\subsection{Non-dimensionalisation}

We non-dimensionalise the governing equations by the dominant scales of the respective physical quantities and name the ratios of a few of them, so as to perform an order of magnitude analysis later.

$$
\begin{aligned}
& (s, \phi, n)=(L \hat{s}, \hat{\phi}, D \hat{n}) ; \\
& \left(\tau, F^{s}, t\right)=\left(\hat{\tau} L, D \hat{F}^{s},(L / g)^{1 / 2} \hat{t}\right) ; \\
& (u, v, w, \Omega)=(g L)^{1 / 2}(\hat{u}, \hat{v}, \epsilon \hat{w}, \hat{\Omega} / L) ; \\
& \left(p_{s s}, p_{\phi \phi}, p_{n n}\right)=\rho_{\circ} g D\left(\hat{p_{s s}}, \hat{p_{\phi \phi}}, \hat{p_{n n}}\right) ; \\
& \left(p_{s \phi}, p_{n \phi}, p_{s n}\right)=\rho_{\circ} g D \mu\left(\hat{p_{s \phi}}, \hat{p_{n \phi}}, \hat{p_{s n}}\right) ;
\end{aligned}
$$

where $\mu=\tan \delta$ in terms of the basal friction angle $\delta, g$ is the maximum magnitude of gravity, $D$ represents the scale for the depth of the flow and $\epsilon=D / L$, is a very small parameter that measures the flow's shallowness. For small $\epsilon$ we simplify the governing equations by averaging them through the depth of the flow.

\subsection{Boundary conditions (BCs), constitutive relations and final equations}

At the top surface of the flow, the kinematic and kinetic conditions applied are the free surface and zero traction BCs respectively. The same at the bottom are the no penetration condition and the Coulomb's law for dry friction.

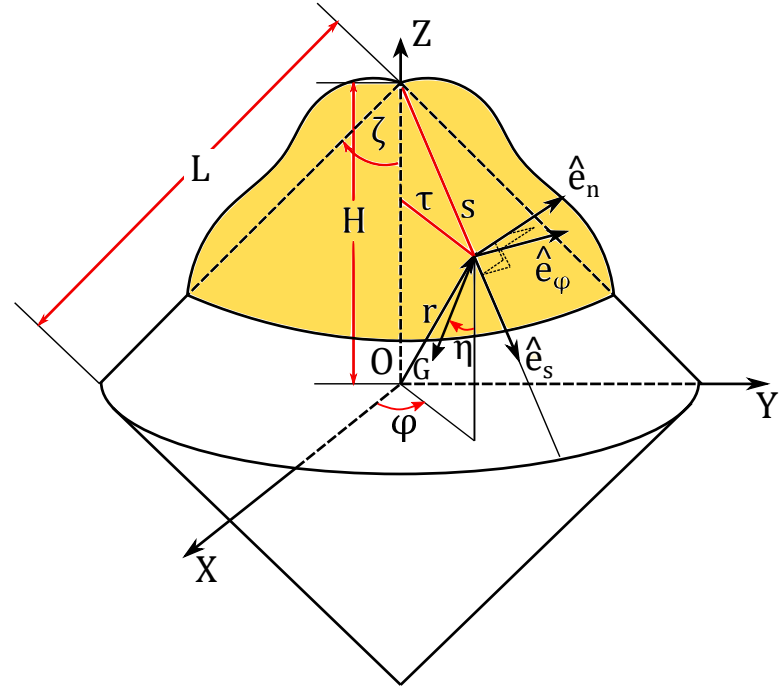

Figure 2. Schematic of our model of a top-shaped asteroid. Here, $\phi$ represents the azimuthal angle and $\hat{\boldsymbol{e}}_{\boldsymbol{s}}, \hat{\boldsymbol{e}}_{n}$ and $\hat{\boldsymbol{e}}_{\phi}$ represent the orthogonal basis vectors of the conical coordinate system.

The basal pressure $p_{n n}^{b}$ is obtained from the momentum balance in the $n$-direction:

$$
p_{n n}^{b}=-h\left(\cos \zeta \frac{v^{2}}{\tau}+2 v \Omega \cos \zeta+a_{n}\right)=: h \psi .
$$

The contribution to the basal pressure are made by the surface curvature, the Coriolis acceleration and the effective gravity, which includes the centrifugal acceleration.

The constitutive law for granular materials appears in the expression for the depth-averaged pressure in the downslope direction:

$$
\overline{p_{s s}}=\frac{1}{2} p_{n n}^{b} K_{s}=\frac{1}{2} \psi h K_{s}
$$

where $K_{s}$ is the Earth pressure coefficient which is a function of basal friction angle $\delta$ and internal friction angle $\theta$ [6]. For simplicity, we have assumed $\delta=\theta$.

Following the procedure outlined in [4] we depth average equations 1 and 2, followed by an order of magnitude analysis and finally incorporate the BCs and the constitutive relations. The mass balance equation thus becomes

$$
\frac{\partial h \tau}{\partial t}+\frac{\partial h \bar{u} \tau}{\partial s}=0
$$

and the downslope and the azimuthal momentum balance equations are,

$$
\begin{array}{r}
\frac{\partial h \bar{u} \tau}{\partial t}+\frac{\partial h \bar{u}^{2} \tau}{\partial s}-h \bar{v}^{2} \sin \zeta=-\left[\varepsilon \frac{\partial h \overline{p_{s s}} \tau}{\partial s}\right. \\
+\operatorname{sgn}(\bar{u}) \tau \psi h \tan \delta]+\tau h\left[2 \Omega \bar{v} \sin \zeta+a_{s}\right] \\
\frac{\partial h \bar{v} \tau}{\partial t}+\frac{\partial h \overline{u v} \tau}{\partial s}+h \overline{u v} \sin \zeta=-\operatorname{sgn}(\bar{v}) \tau \psi h \tan \delta \\
+\tau h[-2 \Omega \bar{u} \sin \zeta+\tau \alpha]
\end{array}
$$

Here, $\bar{u}$ and $\bar{v}$ are the depth-averaged downslope and azimuthal velocities. 


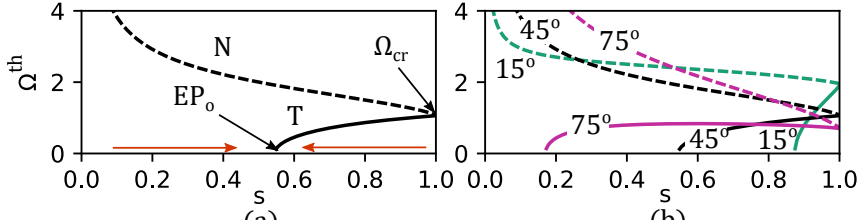

(a)

(b)

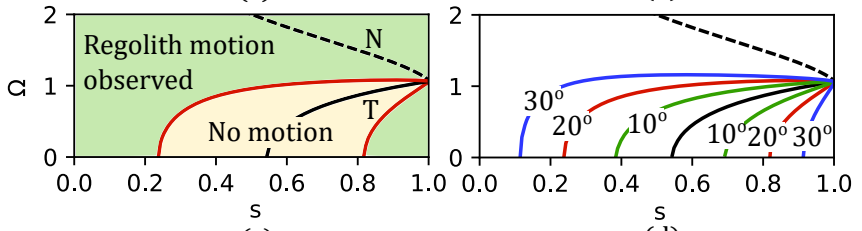

(c)

(d)

Figure 3. (a) Variation with downslope distance of the spin rates $\Omega_{t h}^{s}$ (curve T) and $\Omega_{t h}^{n}$ (curve N) vanishes for (a) $\zeta=45^{\circ}$ and (b) for several $\zeta$. The red arrows in (a) denote the direction of $a_{s}$ on the surface, which changes as one crosses the EP. Regions in the parameter space defined by $\Omega$ and $s$ where regolith can remain immobile for (c) $\delta=20^{\circ}$ bounded by $\Omega_{y}^{ \pm}$(Sec. 3.1) and for (d) different values of $\delta$.

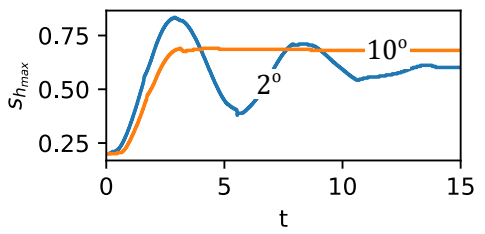

Figure 4. Evolution of the location of maximum height with time for $\Omega_{i}=0.5$ showing how the dune moves for friction angles $\delta=2^{\circ}$ and $10^{\circ}$.

\section{Results and discussion}

Before moving to solve the equations we carry out a preliminary analysis for the behaviour of regolith which is initially stationary with respect to the central body.

\section{1 equilibration and shedding points; static and mobile regions}

On setting $a_{s}$ and $a_{n}$ to zero we obtain the spin rates $\Omega_{s}^{\text {th }}(s)$ and $\Omega_{n}^{\text {th }}(s)$ at which the downslope and normal components of effective gravity change sign, respectively, at a given downslope location $s$. Figure 3 a plots $\Omega_{s}^{t h}$ and $\Omega_{n}^{t h}$ for $\zeta=45^{\circ}$. We call the corresponding downslope locations as the equilibration point (EP) and the shedding point (SP) respectively. There are two important things to note. First, there exists a downslope coordinate at which the effective downslope gravity reverses direction for zero spin rate. This location labelled $\mathrm{EP}_{\circ}$, varies with the semi-apex angle $\zeta$ of the top (Fig. 3b). Second, with an increase in the spin rate the EP shifts downslope towards the equator. The normal acceleration $a_{n}$ vanishes at $\Omega=\Omega_{t h}^{s}(s=1)=\Omega_{c r}$ with the SP occurring at the equator. Hereafter, $\Omega_{n}^{\text {th }}$ increases asymptotically to infinity while the SP moves upslope as seen in Fig. 3a. We note that the $\mathrm{T}$ and $\mathrm{N}$ curves meet at the equator $(s=1)$. Finally, while the vanishing of $a_{n}$ clearly relates to the shedding of grains from the

\begin{tabular}{c|ccc|c}
$\Omega_{i}$ & $s_{i}=0.2$ & $s_{i}=0.5$ & $s_{i}=0.8$ & $\delta$ \\
\hline 0.1 & 0.56 & 0.49 & 0.58 & $10^{\circ}$ \\
0.4 & 0.68 & 0.485 & 0.695 & $10^{\circ}$ \\
0.9 & 0.735 & 0.765 & 0.79 & $10^{\circ}$ \\
1.5 & mass shed & mass shed & mass shed & $10^{\circ}$ \\
0.1 & 0.185 & 0.495 & 0.79 & $30^{\circ}$ \\
0.4 & 0.185 & 0.485 & 0.79 & $30^{\circ}$ \\
0.9 & 0.2 & 0.49 & 0.795 & $30^{\circ}$ \\
1.5 & mass shed & mass shed & mass shed & $30^{\circ}$ \\
\hline
\end{tabular}

Table 1. Location of maximum height $\left(s_{h_{\max }}\right)$ of the final deposit for different initial spin rates $\Omega_{i}$, friction angles $\delta$ and initial dune location $s_{i}$.

body's surface, the significance of $a_{s}$ reversing sign may be understood by considering regolith of no friction.

We now investigate flow initiation in frictional regolith. The basal yield criterion reminiscent of the Coulomb friction law is used to check whether regolith with friction angle $\delta$ remains stationary or starts flowing. For a single stationary grain it may be restated as,

$$
\left|a_{s}\right|>(\tan \delta)\left|a_{n}\right|
$$

By equating the effective downslope gravity and the basal friction in (12), we obtain the maximum and minimum spin rates $\Omega_{y}^{ \pm}$, between which regolith remains stationary at a particular downslope location:

$$
\Omega_{y}^{ \pm}=\sqrt{\frac{ \pm G_{s}-\mu G_{n}}{\tau(\mu \sin \zeta-\cos \zeta)}},
$$

where $G_{s}$ and $G_{n}$ are the magnitudes of the downslope and normal components of gravity. Figure $3 \mathrm{c}$ plots $\Omega_{y}^{ \pm}$versus downslope distance $s$ for $\delta=20^{\circ}$. The region enclosed by the red lines and the horizontal axis represents combinations of spin rate $\Omega$ and downslope location $s$ at which regolith may remain immobile. The EP for a given $\Omega$ always lies within this region. As the friction angle $\delta$ increases, greater regions of the parameter space are occupied by the immobile regolith. This is shown in Fig. 3d. We also notice that for spin rates above $\Omega_{c r}$ the regolith fails at all locations and moves towards the equator from where it is shed.

\subsection{Numerical method}

We solve (9)-(11) employing the central schemes developed in a finite volume framework for hyperbolic conservation laws [7]. We verify our code against solved cases like the dam-break and ensure that shocks are accurately captured. We note that for our system to remain hyperbolic requires that $\psi$ and hence $p_{n n}$ must be positive. Recall that grain shedding takes place when $p_{n n}<0$ at which point regolith is lost from the surface.

\subsection{Landslides and mass shedding}

We now investigate the run-out distances of landslides that originate at different latitudes on the body's surface due to 


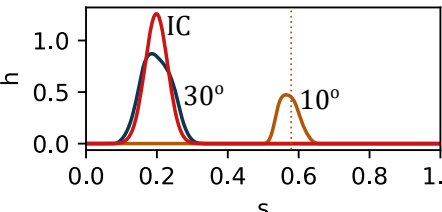

(a)

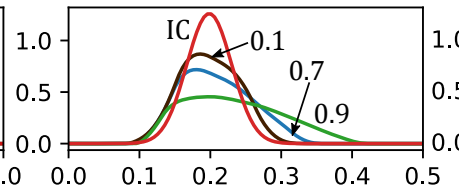

(b)

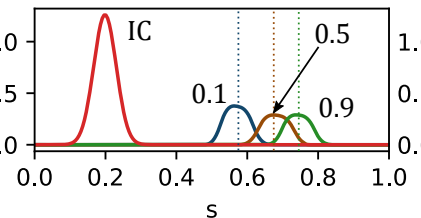

(c)

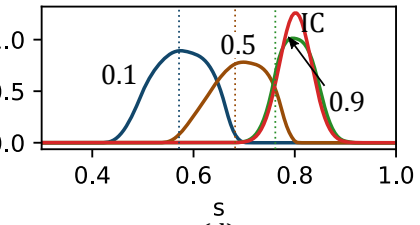

(d)

Figure 5. Final deposit of dunes under different conditions. (a) Different values of friction angle $\delta\left(\Omega_{i}=0.1\right)$. (b) Different $\Omega_{i}$ $\left(\delta=30^{\circ}\right)$. (c) Different $\Omega_{i}\left(\delta=10^{\circ}\right)$. The initial dune is near the pole $(s=0.2)$. (d) Upslope flow for $\delta=10^{\circ}$ at different $\Omega_{i}$ from low latitudes $(s=0.8)$. The dotted vertical lines represent the location of EP for the corresponding spin rate.

(a)

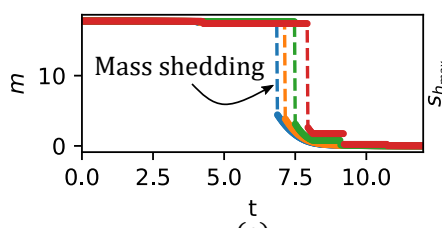

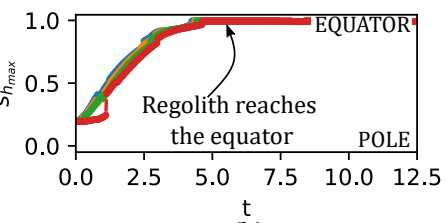

(b)
Figure 6. Plot for different $\Omega_{i}$ (blue - 2.4, orange - 2.1, green 1.8 , red - 1.5) of (a) the total mass of regolith on the surface and (b) the location of maximum height of a dune, with time. The initial dune is at $s=0.2$ as in Fig. 5a and $\delta=30^{\circ}$.

the failure of a stationary mass of regolith or dune. We shall consider the initial condition to be $u=0$ and $v=0$ in the rotating frame and dunes to have Gaussian profiles.

Table 1 shows the location of the maximum height of the final deposit for dunes initially at different latitudes for low and high friction regolith respectively. As expected, regolith of high friction does not move much from it's original location. Significant motion is observed for low friction regolith in the direction of the corresponding EP. Mass is shed completely from the equator at high spin rates; see Fig. 6b.

Figure 5a shows that for $\delta=10^{\circ}$ a near-polar dune $(s=0.2)$ reaches close to the EP which is shown by the dotted line. At even lower $\delta\left(=2^{\circ}\right)$ the dune oscillates about the EP; see Fig. 4. It is to be noted that the maximum height of the dune reduces with downslope motion. This is because the dune spreads over a larger area as it moves towards the equator. In Fig. $5 \mathrm{~b}$ we see the final deposits of the same initial dune for high friction angles. As the spin rate is increased the dune spreads over a greater latitudinal extent but does not shift completely to a new position. Figures $5 \mathrm{c}$ and $\mathrm{d}$ show that low friction near-polar $(s=0.2)$ and near-equatorial ( $s=0.8$ ) dunes move downslope and upslope respectively for the given spin rates. A near-polar dune will never move upslope because the EP for zero spin lies in the mid-latitudes $(s=0.537)$ and shifts towards the equator with increasing spin rate. However, a nearequatorial dune will show upslope and downslope motion for low and high spin rates respectively.

Figure 6 shows that the mass of regolith on the surface lowers, after the dune that started from $s=0.2$ travels to the equator (Fig. 6b), piles up there and finally sheds at time around $t=7.5$ (Fig. 6a). For all spin rates near $\Omega_{c r}=0.176$ mass is always shed from the equator $(s=1)$. The shedding is abrupt because regolith accumulates at the equator and the height is set to zero just as $p_{n n}<0$ is satisfied.

\section{Conclusions}

We have studied shallow axisymmetric granular flows over rotating and gravitating top-shaped asteroids using the theory of avalanche dynamics. The set of onedimensional hyperbolic conservation laws in variables of height, downslope and azimuthal velocities are solved using the central schemes in finite volume methods. The equilibration point EP on the surface is the location where effective tangential gravity vanishes. The shedding point $\mathrm{SP}$ is the location where effective normal gravity vanishes. For a stationary frictionless grain the EP moves downslope with increasing spin rate till it reaches the equator at $\Omega=\Omega_{c r}$. At this spin rate the EP and SP coincide. As $\Omega$ increases further, the SP moves upslope and reaches the pole asymptotically at infinite spin rate. The greater the semi-apex angle of the top, the closer to the pole is the EP。 (i.e. the EP for zero spin rate) and the lower is the $\Omega_{c r}$. For frictional grains, the EP always lies within the static zone. Beyond $\Omega_{c r}$ regolith is always shed. When a mass of regolith (dune) moves downslope, it spreads and the height decreases. Regolith motion is inhibited at higher friction angles. For low friction angles regolith oscillates about the EP. Regolith can never travel upslope of EP . Mass shedding takes place from the equator.

\section{References}

[1] D. S. Lauretta, et al., Nature 568(7750), 55-60 (2019)

[2] P. Paolicchi, et al., Asteroids III.1, 517-26 (2002)

[3] K. Gaurav, et al., J. Fluid. Mech. Accepted. (2021)

[4] J.M.N.T. Gray, et. al. Proc. R. Soc. 1985, 1841-1874 (1999)

[5] D. Banik, Masters Thesis. IIT Kanpur (2020)

[6] R. M. Nedderman, Statics and Kinematics of Granular Materials, Cambridge University Press. (2005)

[7] Kurganov, E., et. al. J. Comput. Phys. 160, 241-282 (2000) 